\title{
A more accurate half-discrete Hilbert-type inequality with a non-homogeneous kernel
}

Bicheng Yang ${ }^{1 *}$ and Xindong Liu²

"Correspondence:
bcyang@gdei.edu.cn
1Department of Mathematics,
Guangdong University of
Education, Guangzhou, Guangdong
510303, China
Full list of author information is
available at the end of the article

available at the end of the article

\begin{abstract}
By means of weight functions and the improved Euler-Maclaurin summation formula, a more accurate half-discrete Hilbert-type inequality with a non-homogeneous kernel and a best constant factor is given. A best extension, some equivalent forms, the operator expressions as well as some particular cases are also considered.
\end{abstract}

MSC: 26D15; 47A07

Keywords: Hilbert-type inequality; weight function; equivalent form; operator

\section{Introduction}

Assuming that $f, g \in L^{2}\left(\mathbf{R}_{+}\right),\|f\|=\left\{\int_{0}^{\infty} f^{2}(x) d x\right\}^{\frac{1}{2}}>0,\|g\|>0$, we have the following Hilbert's integral inequality (cf. [1]):

$$
\int_{0}^{\infty} \int_{0}^{\infty} \frac{f(x) g(y)}{x+y} d x d y<\pi\|f\|\|g\|
$$

where the constant factor $\pi$ is best possible. If $a=\left\{a_{n}\right\}_{n=1}^{\infty}, b=\left\{b_{n}\right\}_{n=1}^{\infty} \in l^{2},\|a\|=$ $\left\{\sum_{n=1}^{\infty} a_{n}^{2}\right\}^{\frac{1}{2}}>0,\|b\|>0$, then we have the following analogous discrete Hilbert's inequality:

$$
\sum_{m=1}^{\infty} \sum_{n=1}^{\infty} \frac{a_{m} b_{n}}{m+n}<\pi\|a\|\|b\|
$$

with the same best constant factor $\pi$. Inequalities (1) and (2) are important in analysis and its applications (cf. [2-4]).

In 1998, by introducing an independent parameter $\lambda \in(0,1]$, Yang [5] gave an extension of (1). For generalizing the results from [5], Yang [6] gave some best extensions of (1) and (2) as follows. If $p>1, \frac{1}{p}+\frac{1}{q}=1, \lambda_{1}+\lambda_{2}=\lambda, k_{\lambda}(x, y)$ is a non-negative homogeneous function of degree $-\lambda$ satisfying $k\left(\lambda_{1}\right)=\int_{0}^{\infty} k_{\lambda}(t, 1) t^{\lambda_{1}-1} d t \in \mathbf{R}_{+}, \phi(x)=x^{p\left(1-\lambda_{1}\right)-1}, \psi(x)=x^{q\left(1-\lambda_{2}\right)-1}$, $f(\geq 0) \in L_{p, \phi}\left(\mathbf{R}_{+}\right)=\left\{f \mid\|f\|_{p, \phi}:=\left\{\int_{0}^{\infty} \phi(x)|f(x)|^{p} d x\right\}^{\frac{1}{p}}<\infty\right\}, g(\geq 0) \in L_{q, \psi}\left(\mathbf{R}_{+}\right)$, and $\|f\|_{p, \phi}$, $\|g\|_{q, \psi}>0$, then

$$
\int_{0}^{\infty} \int_{0}^{\infty} k_{\lambda}(x, y) f(x) g(y) d x d y<k\left(\lambda_{1}\right)\|f\|_{p, \phi}\|g\|_{q, \psi},
$$

where the constant factor $k\left(\lambda_{1}\right)$ is best possible. Moreover, if $k_{\lambda}(x, y)$ is finite and $k_{\lambda}(x, y) x^{\lambda_{1}-1}\left(k_{\lambda}(x, y) y^{\lambda_{2}-1}\right)$ is decreasing for $x>0(y>0)$, then for $a_{m}, b_{n} \geq 0, a=\left\{a_{m}\right\}_{m=1}^{\infty} \in$

\section{Springer}

(0) 2012 Yang and Liu; licensee Springer. This is an Open Access article distributed under the terms of the Creative Commons Attribution License (http://creativecommons.org/licenses/by/2.0), which permits unrestricted use, distribution, and reproduction in any medium, provided the original work is properly cited. 
$l_{p, \phi}=\left\{a \mid\|a\|_{p, \phi}:=\left\{\sum_{n=1}^{\infty} \phi(n)\left|a_{n}\right|^{p}\right\}^{\frac{1}{p}}<\infty\right\}$, and $b=\left\{b_{n}\right\}_{n=1}^{\infty} \in l_{q, \psi},\|a\|_{p, \phi},\|b\|_{q, \psi}>0$, we have

$$
\sum_{m=1}^{\infty} \sum_{n=1}^{\infty} k_{\lambda}(m, n) a_{m} b_{n}<k\left(\lambda_{1}\right)\|a\|_{p, \phi}\|b\|_{q, \psi}
$$

where the constant $k\left(\lambda_{1}\right)$ is still the best value. Clearly, for $p=q=2, \lambda=1, k_{1}(x, y)=\frac{1}{x+y}$, $\lambda_{1}=\lambda_{2}=\frac{1}{2}$, (3) reduces to (1), while (4) reduces to (2).

Some other results about integral and discrete Hilbert-type inequalities can be found in [7-16]. On half-discrete Hilbert-type inequalities with the general non-homogeneous kernels, Hardy et al. provided a few results in Theorem 351 of [1]. But they did not prove that the constant factors are best possible. In 2005, Yang [17] gave a result with the kernel $\frac{1}{(1+n x)^{\lambda}}$ by introducing a variable and proved that the constant factor is best possible. Very recently, Yang [18] and [19] gave the following half-discrete Hilbert's inequality with the best constant factor:

$$
\int_{0}^{\infty} f(x) \sum_{n=1}^{\infty} \frac{a_{n}}{(x+n)^{\lambda}} d x<\pi\|f\|\|a\| ;
$$

Chen [20] and Yang [21] gave two more accurate half-discrete Mulholland's inequalities by using Hadamard's inequality.

In this paper, by means of weight functions and the improved Euler-Maclaurin summation formula, a more accurate half-discrete Hilbert-type inequality with a nonhomogeneous kernel and a best constant factor is given as follows. For $0<\alpha+\beta \leq 2$, $\gamma \in \mathbf{R}, \eta \leq 1-\frac{\alpha+\beta}{8}\left(1+\sqrt{3+\frac{4}{\alpha+\beta}}\right)$

$$
\begin{aligned}
& \int_{\gamma}^{\infty} f(x) \sum_{n=1}^{\infty} \frac{(\min \{1,(x-\gamma)(n-\eta)\})^{\beta}}{(\max \{1,(x-\gamma)(n-\eta)\})^{\alpha}} a_{n} d x \\
& \quad<\frac{4}{\alpha+\beta}\left\{\int_{\gamma}^{\infty}(x-\gamma)^{1-\alpha+\beta} f^{2}(x) d x \sum_{n=1}^{\infty}(n-\eta)^{1-\alpha+\beta} a_{n}^{2}\right\}^{\frac{1}{2}} .
\end{aligned}
$$

Moreover, a best extension of (6), some equivalent forms, the operator expressions as well as some particular inequalities are considered.

\section{Some lemmas}

Lemma 1 If $n_{0} \in \mathbf{N}, s>n_{0}, g_{1}(y)\left(y \in\left[n_{0}, s\right)\right), g_{2}(y)(y \in[s, \infty))$ are decreasing continuous functions satisfying $g_{1}\left(n_{0}\right)-g_{1}(s-0)+g_{2}(s)>0, g_{2}(\infty)=0$, define a function $g(y)$ as follows:

$$
g(y):= \begin{cases}g_{1}(y), & y \in\left[n_{0}, s\right) \\ g_{2}(y), & y \in[s, \infty)\end{cases}
$$

Then there exists $\varepsilon \in[0,1]$ such that

$$
\begin{aligned}
& \frac{-1}{8}\left[g_{1}\left(n_{0}\right)+\varepsilon\left(g_{2}(s)-g_{1}(s-0)\right)\right] \\
& <\int_{n_{0}}^{\infty} \rho(y) g(y) d y<\frac{1-\varepsilon}{8}\left(g_{2}(s)-g_{1}(s-0)\right),
\end{aligned}
$$


where $\rho(y)=y-[y]-\frac{1}{2}$ is a Bernoulli function of the first order. In particular, for $g_{1}(y)=0$, $y \in\left[n_{0}, s\right)$, we have $g_{2}(s)>0$ and

$$
\frac{-1}{8} g_{2}(s)<\int_{s}^{\infty} \rho(y) g(y) d y<\frac{1}{8} g_{2}(s)
$$

for $g_{2}(y)=0, y \in[s, \infty)$, if $g_{1}(s-0) \geq 0$, then it follows $g_{1}\left(n_{0}\right)>0$ and

$$
\frac{-1}{8} g_{1}\left(n_{0}\right)<\int_{n_{0}}^{s} \rho(y) g_{1}(y) d y<0 .
$$

Proof Define a decreasing continuous function $\widetilde{g}(y)$ as

$$
\widetilde{g}(y):= \begin{cases}g_{1}(y)+g_{2}(s)-g_{1}(s-0), & y \in\left[n_{0}, s\right), \\ g_{2}(y), & y \in[s, \infty)\end{cases}
$$

Then it follows

$$
\begin{aligned}
& \int_{n_{0}}^{\infty} \rho(y) g(y) d y=\int_{n_{0}}^{s} \rho(y) g(y) d y+\int_{s}^{\infty} \rho(y) g(y) d y \\
& =\int_{n_{0}}^{s} \rho(y)\left(\widetilde{g}(y)-g_{2}(s)+g_{1}(s-0)\right) d y+\int_{s}^{\infty} \rho(y) \widetilde{g}(y) d y \\
& =\int_{n_{0}}^{\infty} \rho(y) \widetilde{g}(y) d y-\left(g_{2}(s)-g_{1}(s-0)\right) \int_{n_{0}}^{s} \rho(y) d y, \\
& \int_{n_{0}}^{s} \rho(y) d y=\int_{n_{0}}^{[s]} \rho(y) d y+\int_{[s]}^{s} \rho(y) d y=\int_{[s]}^{s}\left(y-[s]-\frac{1}{2}\right) d y \\
& =\frac{1}{8}\left[4\left(s-[s]-\frac{1}{2}\right)^{2}-1\right]=\frac{\varepsilon-1}{8} \quad(\varepsilon \in[0,1]) \text {. }
\end{aligned}
$$

Since $\widetilde{g}\left(n_{0}\right)=g_{1}\left(n_{0}\right)+g_{2}(s)-g_{1}(s-0)>0, \tilde{g}(y)$ is a non-constant decreasing continuous function with $\widetilde{g}(\infty)=g_{2}(\infty)=0$, by the improved Euler-Maclaurin summation formula (cf. [6], Theorem 2.2.2), it follows

$$
\frac{-1}{8}\left(g_{1}\left(n_{0}\right)+g_{2}(s)-g_{1}(s-0)\right)=\frac{-1}{8} \widetilde{g}\left(n_{0}\right)<\int_{n_{0}}^{\infty} \rho(y) \widetilde{g}(y) d y<0,
$$

and then in view of the above results and by simple calculation, we have (7).

Lemma 2 If $0<\alpha+\beta \leq 2, \gamma \in \mathbf{R}, \eta \leq 1-\frac{\alpha+\beta}{8}\left(1+\sqrt{3+\frac{4}{\alpha+\beta}}\right)$, and $\omega(n)$ and $\varpi(x)$ are weight functions given by

$$
\begin{aligned}
& \omega(n):=\int_{\gamma}^{\infty} \frac{(\min \{1,(x-\gamma)(n-\eta)\})^{\beta}}{(\max \{1,(x-\gamma)(n-\eta)\})^{\alpha}} \frac{(n-\eta)^{\frac{\alpha-\beta}{2}}}{(x-\gamma)^{1-\frac{\alpha-\beta}{2}}} d x, \quad n \in \mathbf{N}, \\
& \varpi(x):=\sum_{n=1}^{\infty} \frac{(\min \{1,(x-\gamma)(n-\eta)\})^{\beta}}{(\max \{1,(x-\gamma)(n-\eta)\})^{\alpha}} \frac{(x-\gamma)^{\frac{\alpha-\beta}{2}}}{(n-\eta)^{1-\frac{\alpha-\beta}{2}}}, \quad x>\gamma,
\end{aligned}
$$


then we have

$$
\varpi(x)<\omega(n)=\frac{4}{\alpha+\beta} .
$$

Proof Substituting $t=(x-\gamma)(n-\eta)$ in (10), and by simple calculation, we have

$$
\begin{aligned}
\omega(n) & =\int_{0}^{\infty} \frac{(\min \{1, t\})^{\beta}}{(\max \{1, t\})^{\alpha}} t^{\frac{\alpha-\beta}{2}-1} d t \\
& =\int_{0}^{1} t^{\beta+\frac{\alpha-\beta}{2}-1} d t+\int_{1}^{\infty} t^{-\alpha+\frac{\alpha-\beta}{2}-1} d t=\frac{4}{\alpha+\beta} .
\end{aligned}
$$

For fixed $x>\gamma$, we find

$$
\begin{aligned}
& h(x, y):=(x-\gamma)^{\frac{\alpha-\beta}{2}} \frac{(\min \{1,(x-\gamma)(y-\eta)\})^{\beta}}{(\max \{1,(x-\gamma)(y-\eta)\})^{\alpha}}(y-\eta)^{\frac{\alpha-\beta}{2}-1} \\
&= \begin{cases}(x-\gamma)^{\frac{\alpha+\beta}{2}}(y-\eta)^{\frac{\alpha+\beta}{2}}-1, & \eta<y<\eta+\frac{1}{x-\gamma}, \\
(x-\gamma)^{-\frac{\alpha+\beta}{2}}(y-\eta)^{-\frac{\alpha+\beta}{2}-1}, & y \geq \eta+\frac{1}{x-\gamma},\end{cases} \\
& h_{y}^{\prime}(x, y)= \begin{cases}-\left(1-\frac{\alpha+\beta}{2}\right)(x-\gamma)^{\frac{\alpha+\beta}{2}}(y-\eta)^{\frac{\alpha+\beta}{2}-2}, & \eta<y<\eta+\frac{1}{x-\gamma}, \\
-\left(\frac{\alpha+\beta}{2}+1\right)(x-\gamma)^{-\frac{\alpha+\beta}{2}}(y-\eta)^{-\frac{\alpha+\beta}{2}-2}, & y \geq \eta+\frac{1}{x-\gamma},\end{cases} \\
& \int_{\eta}^{\infty} h(x, y) d y \stackrel{t=(x-\gamma)(y-\eta)}{=} \int_{0}^{\infty} \frac{(\min \{1, t\})^{\beta}}{(\max \{1, t\})^{\alpha}} t^{\frac{\alpha-\beta}{2}-1} d t=\frac{4}{\alpha+\beta} .
\end{aligned}
$$

By the Euler-Maclaurin summation formula (cf. [6]), it follows

$$
\begin{aligned}
\varpi(x) & =\sum_{n=1}^{\infty} h(x, n)=\int_{1}^{\infty} h(x, y) d y+\frac{1}{2} h(x, 1)+\int_{1}^{\infty} \rho(y) h_{y}^{\prime}(x, y) d y \\
& =\int_{\eta}^{\infty} h(x, y) d y-R(x)=\frac{4}{\alpha+\beta}-R(x), \\
R(x) & :=\int_{\eta}^{1} h(x, y) d y-\frac{1}{2} h(x, 1)-\int_{1}^{\infty} \rho(y) h_{y}^{\prime}(x, y) d y .
\end{aligned}
$$

(i) For $0<x-\gamma<\frac{1}{1-\eta}$, we obtain $-\frac{1}{2} h(x, 1)=-\frac{1}{2}(x-\gamma)^{\frac{\alpha+\beta}{2}}(1-\eta)^{\frac{\alpha+\beta}{2}-1}$, and

$$
\int_{\eta}^{1} h(x, y) d y=(x-\gamma)^{\frac{\alpha+\beta}{2}} \int_{\eta}^{1}(y-\eta)^{\frac{\alpha+\beta}{2}-1} d y=\frac{2(1-\eta)^{\frac{\alpha+\beta}{2}}}{\alpha+\beta}(x-\gamma)^{\frac{\alpha+\beta}{2}} .
$$

Setting $g(y):=-h_{y}^{\prime}(x, y)$, wherefrom $g_{1}(y)=\left(1-\frac{\alpha+\beta}{2}\right)(x-\gamma)^{\frac{\alpha+\beta}{2}}(y-\eta)^{\frac{\alpha+\beta}{2}-2}, g_{2}(y)=$ $\left(\frac{\alpha+\beta}{2}+1\right)(x-\gamma)^{-\frac{\alpha+\beta}{2}}(y-\eta)^{-\frac{\alpha+\beta}{2}-2}$ and

$$
\begin{aligned}
& g_{2}\left(\eta+\frac{1}{x-\gamma}\right)-g_{1}\left(\left(\eta+\frac{1}{x-\gamma}\right)-0\right) \\
& \quad=\left(\frac{\alpha+\beta}{2}+1\right)(x-\gamma)^{2}-\left(1-\frac{\alpha+\beta}{2}\right)(x-\gamma)^{2} \\
& \quad=(\alpha+\beta)(x-\gamma)^{2}>0,
\end{aligned}
$$


then by (7), we find

$$
\begin{aligned}
-\int_{1}^{\infty} \rho(y) h_{y}^{\prime}(x, y) d y= & \int_{1}^{\infty} \rho(y) g(y) d y \\
> & \frac{-1}{8}\left[g_{1}(1)+g_{2}\left(\eta+\frac{1}{x-\gamma}\right)-g_{1}\left(\left(\eta+\frac{1}{x-\gamma}\right)-0\right)\right] \\
= & \frac{-1}{8}\left[\left(1-\frac{\alpha+\beta}{2}\right)(x-\gamma)^{\frac{\alpha+\beta}{2}}(1-\eta)^{\frac{\alpha+\beta}{2}-2}+(\alpha+\beta)(x-\gamma)^{2}\right] \\
> & \frac{-1}{8}\left[\left(1-\frac{\alpha+\beta}{2}\right)(1-\eta)^{\frac{\alpha+\beta}{2}-2}(x-\gamma)^{\frac{\alpha+\beta}{2}}\right. \\
& \left.+(\alpha+\beta)(1-\eta)^{\frac{\alpha+\beta}{2}-2}(x-\gamma)^{\frac{\alpha+\beta}{2}-2}(x-\gamma)^{2}\right] \\
= & \frac{-1}{8}\left[\left(1+\frac{\alpha+\beta}{2}\right)(1-\eta)^{\frac{\alpha+\beta}{2}-2}(x-\gamma)^{\frac{\alpha+\beta}{2}}\right] .
\end{aligned}
$$

In view of (11) and the above results, since for $\eta \leq 1-\frac{\alpha+\beta}{8}\left(1+\sqrt{3+\frac{4}{\alpha+\beta}}\right)$, namely $1-\eta \geq$ $\frac{\alpha+\beta}{8}\left(1+\sqrt{3+\frac{4}{\alpha+\beta}}\right)$, it follows

$$
\begin{aligned}
R(x)> & \frac{2}{\alpha+\beta}(1-\eta)^{\frac{\alpha+\beta}{2}}(x-\gamma)^{\frac{\alpha+\beta}{2}}-\frac{1}{2}(x-\gamma)^{\frac{\alpha+\beta}{2}}(1-\eta)^{\frac{\alpha+\beta}{2}-1} \\
& -\frac{1}{8}\left(1+\frac{\alpha+\beta}{2}\right)(1-\eta)^{\frac{\alpha+\beta}{2}}-2(x-\gamma)^{\frac{\alpha+\beta}{2}} \\
= & {\left[\frac{2(1-\eta)^{2}}{\alpha+\beta}-\frac{(1-\eta)}{2}-\frac{2+\alpha+\beta}{16}\right] \frac{(x-\gamma)^{\frac{\alpha+\beta}{2}}}{(1-\eta)^{2-\frac{\alpha+\beta}{2}}} \geq 0 . }
\end{aligned}
$$

(ii) For $x-\gamma \geq \frac{1}{1-\eta}$, we obtain $-\frac{1}{2} h(x, 1)=-\frac{1}{2}(x-\gamma)^{-\frac{\alpha+\beta}{2}}(1-\eta)^{-\frac{\alpha+\beta}{2}-1}$, and

$$
\begin{aligned}
\int_{\eta}^{1} h(x, y) d y & =\int_{\eta}^{\eta+\frac{1}{x-\gamma}} \frac{(x-\gamma)^{\frac{\alpha+\beta}{2}}}{(y-\eta)^{1-\frac{\alpha+\beta}{2}}} d y+\int_{\eta+\frac{1}{x-\gamma}}^{1} \frac{(x-\gamma)^{-\frac{\alpha+\beta}{2}}}{(y-\eta)^{\frac{\alpha+\beta}{2}+1}} d y \\
& =\frac{4}{\alpha+\beta}-\frac{2}{\alpha+\beta}(1-\eta)^{-\frac{\alpha+\beta}{2}}(x-\gamma)^{-\frac{\alpha+\beta}{2}} \\
& \geq \frac{4(1-\eta)^{-\frac{\alpha+\beta}{2}}}{\alpha+\beta}(x-\gamma)^{-\frac{\alpha+\beta}{2}}-\frac{2(1-\eta)^{-\frac{\alpha+\beta}{2}}}{\alpha+\beta}(x-\gamma)^{-\frac{\alpha+\beta}{2}} \\
& =\frac{2}{\alpha+\beta}(1-\eta)^{-\frac{\alpha+\beta}{2}}(x-\gamma)^{-\frac{\alpha+\beta}{2}}
\end{aligned}
$$

Since for $y \geq 1, y-\eta \geq \frac{1}{x-\gamma}$, by the improved Euler-Maclaurin summation formula (cf. [6]), it follows

$$
\begin{aligned}
-\int_{1}^{\infty} \rho(y) h_{y}^{\prime}(x, y) d y & =\left(\frac{\alpha+\beta}{2}+1\right)(x-\gamma)^{-\frac{\alpha+\beta}{2}} \int_{1}^{\infty} \rho(y)(y-\eta)^{-\frac{\alpha+\beta}{2}-2} d y \\
& >-\frac{1}{12}\left(\frac{\alpha+\beta}{2}+1\right)(x-\gamma)^{-\frac{\alpha+\beta}{2}}(1-\eta)^{-\frac{\alpha+\beta}{2}-2} .
\end{aligned}
$$


In view of (13) and the above results, for $1-\eta \geq \frac{\alpha+\beta}{8}\left(1+\sqrt{3+\frac{4}{\alpha+\beta}}\right)$, we find

$$
\begin{aligned}
R(x)> & \frac{2}{\alpha+\beta}(1-\eta)^{-\frac{\alpha+\beta}{2}}(x-\gamma)^{-\frac{\alpha+\beta}{2}}-\frac{1}{2}(1-\eta)^{-\frac{\alpha+\beta}{2}-1}(x-\gamma)^{-\frac{\alpha+\beta}{2}} \\
& -\frac{1}{12}\left(\frac{\alpha+\beta}{2}+1\right)(1-\eta)^{-\frac{\alpha+\beta}{2}-2}(x-\gamma)^{-\frac{\alpha+\beta}{2}} \\
> & {\left[\frac{2(1-\eta)^{2}}{\alpha+\beta}-\frac{1-\eta}{2}-\frac{2+\alpha+\beta}{16}\right] \frac{(x-\gamma)^{-\frac{\alpha+\beta}{2}}}{(1-\eta)^{2+\frac{\alpha+\beta}{2}}} \geq 0 . }
\end{aligned}
$$

Hence, for $x>\gamma$, we have $R(x)>0$, and then (12) follows.

Lemma 3 Let the assumptions of Lemma 2 be fulfilled and, additionally, let $p>1, \frac{1}{p}+\frac{1}{q}=1$, $a_{n} \geq 0, n \in \mathbf{N}, f(x)$ be a non-negative measurable function in $(\gamma, \infty)$. Then we have the following inequalities:

$$
\begin{aligned}
J & :=\left\{\sum_{n=1}^{\infty}(n-\beta)^{\frac{p(\alpha-\beta)}{2}-1}\left[\int_{\gamma}^{\infty} \frac{(\min \{1,(x-\gamma)(n-\eta)\})^{\beta}}{(\max \{1,(x-\gamma)(n-\eta)\})^{\alpha}} f(x) d x\right]^{p}\right\}^{\frac{1}{p}} \\
& \leq\left(\frac{4}{\alpha+\beta}\right)^{\frac{1}{q}}\left\{\int_{\gamma}^{\infty} \varpi(x)(x-\gamma)^{p\left(1-\frac{\alpha-\beta}{2}\right)-1} f^{p}(x) d x\right\}^{\frac{1}{p}}, \\
L_{1} & :=\left\{\int_{\gamma}^{\infty} \frac{(x-\alpha)^{\frac{q(\alpha-\beta)}{2}}-1}{[\varpi(x)]^{q-1}}\left[\sum_{n=1}^{\infty} \frac{(\min \{1,(x-\gamma)(n-\eta)\})^{\beta} a_{n}}{(\max \{1,(x-\gamma)(n-\eta)\})^{\alpha}}\right]^{q} d x\right\}^{\frac{1}{q}} \\
& \leq\left\{\frac{4}{\alpha+\beta} \sum_{n=1}^{\infty}(n-\eta)^{q\left(1-\frac{\alpha-\beta}{2}\right)-1} a_{n}^{q}\right\}^{\frac{1}{q}} .
\end{aligned}
$$

Proof Setting $k(x, n):=\frac{(\min \{1,(x-\gamma)(n-\eta)\})^{\beta}}{(\max \{1,(x-\gamma)(n-\eta)\})^{\alpha}}$, by Hölder's inequality $(c f .[22])$ and (12), it follows

$$
\begin{aligned}
& {\left[\int_{\gamma}^{\infty} \frac{(\min \{1,(x-\gamma)(n-\eta)\})^{\beta}}{(\max \{1,(x-\gamma)(n-\eta)\})^{\alpha}} f(x) d x\right]^{p}} \\
& =\left\{\int_{\gamma}^{\infty} k(x, n)\left[\frac{(x-\gamma)^{\left(1-\frac{\alpha-\beta}{2}\right) / q}}{(n-\eta)^{\left(1-\frac{\alpha-\beta}{2}\right) / p}} f(x)\right]\left[\frac{(n-\gamma)^{\left(1-\frac{\alpha-\beta}{2}\right) / p}}{(x-\gamma)^{\left(1-\frac{\alpha-\beta}{2}\right) / q}}\right] d x\right\}^{p} \\
& \leq \int_{\gamma}^{\infty} k(x, n) \frac{(x-\gamma)^{\left(1-\frac{\alpha-\beta}{2}\right)(p-1)}}{(n-\eta)^{1-\frac{\alpha-\beta}{2}}} f^{p}(x) d x \\
& \quad \times\left\{\int_{\gamma}^{\infty} k(x, n) \frac{(n-\eta)^{\left(1-\frac{\alpha-\beta}{2}\right)(q-1)}}{(x-\gamma)^{1-\frac{\alpha-\beta}{2}}} d x\right\}^{p-1} \\
& =\left\{\omega(n)(n-\eta)^{q\left(1-\frac{\alpha-\beta}{2}\right)-1}\right\}^{p-1} \int_{\gamma}^{\infty} k(x, n) \frac{(x-\gamma)^{\left(1-\frac{\alpha-\beta}{2}\right)(p-1)}}{(n-\eta)^{1-\frac{\alpha-\beta}{2}}} f^{p}(x) d x \\
& =\left(\frac{4}{\alpha+\beta}\right)^{p-1}(n-\eta)^{1-\frac{p(\alpha-\beta)}{2}} \int_{\gamma}^{\infty} k(x, n) \frac{(x-\gamma)^{\left(1-\frac{\alpha-\beta}{2}\right)(p-1)}}{(n-\eta)^{1-\frac{\alpha-\beta}{2}}} f^{p}(x) d x .
\end{aligned}
$$


Then by the Lebesgue term-by-term integration theorem ( $c f$. [23]), we have

$$
\begin{aligned}
J & \leq\left(\frac{4}{\alpha+\beta}\right)^{\frac{1}{q}}\left\{\sum_{n=1}^{\infty} \int_{\gamma}^{\infty} k(x, n) \frac{(x-\gamma)^{\left(1-\frac{\alpha-\beta}{2}\right)(p-1)}}{(n-\eta)^{1-\frac{\alpha-\beta}{2}}} f^{p}(x) d x\right\}^{\frac{1}{p}} \\
& =\left(\frac{4}{\alpha+\beta}\right)^{\frac{1}{q}}\left\{\int_{\gamma}^{\infty} \sum_{n=1}^{\infty} k(x, n) \frac{(x-\gamma)^{\left(1-\frac{\alpha-\beta}{2}\right)(p-1)}}{(n-\eta)^{1-\frac{\alpha-\beta}{2}}} f^{p}(x) d x\right\}^{\frac{1}{p}} \\
& =\left(\frac{4}{\alpha+\beta}\right)^{\frac{1}{q}}\left\{\int_{\gamma}^{\infty} \varpi(x)(x-\gamma)^{p\left(1-\frac{\alpha-\beta}{2}\right)-1} f^{p}(x) d x\right\}^{\frac{1}{p}} .
\end{aligned}
$$

Hence, (14) follows. By Hölder's inequality again, we have

$$
\begin{aligned}
{\left[\sum_{n=1}^{\infty} k(x, n) a_{n}\right]^{q}=} & \left\{\sum_{n=1}^{\infty} k(x, n)\left[\frac{(x-\gamma)^{\left(1-\frac{\alpha-\beta}{2}\right) / q}}{(n-\eta)^{\left(1-\frac{\alpha-\beta}{2}\right) / p}}\right]\right. \\
& \left.\times\left[\frac{(n-\eta)^{\left(1-\frac{\alpha-\beta}{2}\right) / p} a_{n}}{(x-\gamma)^{\left(1-\frac{\alpha-\beta}{2}\right) / q}}\right]\right\}^{q} \leq\left\{\sum_{n=1}^{\infty} k(x, n) \frac{(x-\gamma)^{\left(1-\frac{\alpha-\beta}{2}\right)(p-1)}}{(n-\eta)^{1-\frac{\alpha-\beta}{2}}}\right\} \\
& \times \sum_{n=1}^{\infty} k(x, n) \frac{(n-\eta)^{\left(1-\frac{\alpha-\beta}{2}\right)(q-1)}}{(x-\gamma)^{1-\frac{\alpha-\beta}{2}}} a_{n}^{q} \\
= & \frac{[\varpi(x)]^{q-1}}{(x-\gamma)^{\frac{q(\alpha-\beta)}{2}}-1} \sum_{n=1}^{\infty} k(x, n) \frac{(n-\eta)^{\left(1-\frac{\alpha-\beta}{2}\right)(q-1)}}{(x-\gamma)^{1-\frac{\alpha-\beta}{2}}} a_{n}^{q} .
\end{aligned}
$$

By the Lebesgue term-by-term integration theorem, we have

$$
\begin{aligned}
L_{1} & \leq\left\{\int_{\gamma}^{\infty} \sum_{n=1}^{\infty} k(x, n) \frac{(n-\eta)^{\left(1-\frac{\alpha-\beta}{2}\right)(q-1)}}{(x-\gamma)^{1-\frac{\alpha-\beta}{2}}} a_{n}^{q} d x\right\}^{\frac{1}{q}} \\
& =\left\{\sum_{n=1}^{\infty} \int_{\gamma}^{\infty} k(x, n) \frac{(n-\eta)^{\left(1-\frac{\alpha-\beta}{2}\right)(q-1)}}{(x-\gamma)^{1-\frac{\alpha-\beta}{2}}} a_{n}^{q} d x\right\}^{\frac{1}{q}} \\
& =\left\{\sum_{n=1}^{\infty} \omega(n)(n-\eta)^{q\left(1-\frac{\alpha-\beta}{2}\right)-1} a_{n}^{q}\right\}^{\frac{1}{q}},
\end{aligned}
$$

and in view of (12), inequality (15) follows.

Lemma 4 Let the assumptions of Lemma 2 be fulfilled and, additionally, let $p>1, \frac{1}{p}+\frac{1}{q}=1$, $0<\varepsilon<\frac{p}{2}(\alpha+\beta)$. Setting $\widetilde{f}(x)=(x-\gamma)^{\frac{\alpha-\beta}{2}+\frac{\varepsilon}{p}-1}, x \in(\gamma, \gamma+1) ; \widetilde{f}(x)=0, x \in[\gamma+1, \infty)$, and $\tilde{a}_{n}=(n-\eta)^{\frac{\alpha-\beta}{2}-\frac{\varepsilon}{q}-1}, n \in \mathbf{N}$, then we have

$$
\begin{aligned}
\widetilde{I} & :=\sum_{n=1}^{\infty} \widetilde{a}_{n} \int_{\gamma}^{\infty} \frac{(\min \{1,(x-\gamma)(n-\eta)\})^{\beta}}{(\max \{1,(x-\gamma)(n-\eta)\})^{\alpha}} \tilde{f}(x) d x \\
& >\frac{1}{\varepsilon}\left[\frac{(\alpha+\beta)(1-\eta)^{-\varepsilon}}{\left(\frac{\alpha+\beta}{2}\right)^{2}-\left(\frac{\varepsilon}{p}\right)^{2}}-\varepsilon O(1)\right],
\end{aligned}
$$




$$
\begin{aligned}
& \widetilde{H}:=\left\{\int_{\gamma}^{\infty}(x-\gamma)^{p\left(1-\frac{\alpha-\beta}{2}\right)-1} \widetilde{f}^{p}(x) d x\right\}^{\frac{1}{p}}\left\{\sum_{n=1}^{\infty}(n-\eta)^{q\left(1-\frac{\alpha-\beta}{2}\right)-1} \widetilde{a}_{n}^{q}\right\}^{\frac{1}{q}} \\
& <\frac{1}{\varepsilon}\left[\varepsilon(1-\eta)^{-\varepsilon-1}+(1-\eta)^{-\varepsilon}\right]^{\frac{1}{q}} .
\end{aligned}
$$

Proof We find

$$
\begin{aligned}
\widetilde{I}= & \sum_{n=1}^{\infty}(n-\eta)^{\frac{\alpha-\beta}{2}-\frac{\varepsilon}{q}-1} \\
& \times \int_{\gamma}^{\gamma+1} \frac{(\min \{1,(x-\gamma)(n-\eta)\})^{\beta}}{(\max \{1,(x-\gamma)(n-\eta)\})^{\alpha}}(x-\gamma)^{\frac{\alpha-\beta}{2}+\frac{\varepsilon}{p}-1} d x \\
= & \sum_{n=1}^{\infty}(n-\eta)^{\frac{\alpha-\beta}{2}-\frac{\varepsilon}{q}-1}\left[(n-\eta)^{\beta} \int_{\gamma}^{\gamma+\frac{1}{n-\eta}}(x-\gamma)^{\frac{\alpha+\beta}{2}+\frac{\varepsilon}{p}-1} d x\right. \\
& \left.+\frac{1}{(n-\eta)^{\alpha}} \int_{\gamma+\frac{1}{n-\eta}}^{\gamma+1}(x-\gamma)^{-\frac{\alpha+\beta}{2}+\frac{\varepsilon}{p}-1} d x\right] \\
= & \frac{\alpha+\beta}{\left(\frac{\alpha+\beta}{2}\right)^{2}-\left(\frac{\varepsilon}{p}\right)^{2}} \sum_{n=1}^{\infty}(n-\eta)^{-\varepsilon-1}-\frac{1}{\frac{\alpha+\beta}{2}-\frac{\varepsilon}{p}} \sum_{n=1}^{\infty}(n-\eta)^{-\frac{\alpha+\beta}{2}-\frac{\varepsilon}{q}-1} \\
> & \frac{\alpha+\beta}{\left(\frac{\alpha+\beta}{2}\right)^{2}-\left(\frac{\varepsilon}{p}\right)^{2}} \int_{1}^{\infty} \frac{d y}{(y-\eta)^{\varepsilon+1}}-\frac{1}{\frac{\alpha+\beta}{2}-\frac{\varepsilon}{p}} \sum_{n=1}^{\infty}(n-\eta)^{-\frac{\alpha+\beta}{2}-\frac{\varepsilon}{q}-1} \\
= & \frac{1}{\varepsilon}\left[\frac{(\alpha+\beta)(1-\eta)^{-\varepsilon}}{\left(\frac{\alpha+\beta}{2}\right)^{2}-\left(\frac{\varepsilon}{p}\right)^{2}}-\frac{\varepsilon}{\frac{\alpha+\beta}{2}-\frac{\varepsilon}{p}} \sum_{n=1}^{\infty}(n-\eta)^{-\frac{\alpha+\beta}{2}-\frac{\varepsilon}{q}-1}\right],
\end{aligned}
$$

and then (16) is valid. We obtain

$$
\begin{aligned}
\widetilde{H} & =\left\{\int_{\gamma}^{\gamma+1}(x-\gamma)^{\varepsilon-1} d x\right\}^{\frac{1}{p}}\left\{(1-\eta)^{-\varepsilon-1}+\sum_{n=2}^{\infty}(n-\eta)^{-\varepsilon-1}\right\}^{\frac{1}{q}} \\
& <\left(\frac{1}{\varepsilon}\right)^{\frac{1}{p}}\left\{(1-\eta)^{-\varepsilon-1}+\int_{1}^{\infty}(y-\eta)^{-\varepsilon-1} d y\right\}^{\frac{1}{q}} \\
& =\frac{1}{\varepsilon}\left\{\varepsilon(1-\eta)^{-\varepsilon-1}+(1-\eta)^{-\varepsilon}\right\}^{\frac{1}{q}},
\end{aligned}
$$

and so (17) is valid.

\section{Main results}

We introduce the functions

$$
\Phi(x):=(x-\gamma)^{p\left(1-\frac{\alpha-\beta}{2}\right)-1} \quad(x \in(\gamma, \infty)), \quad \Psi(n):=(n-\eta)^{q\left(1-\frac{\alpha-\beta}{2}\right)-1} \quad(n \in \mathbf{N}),
$$

wherefrom $[\Phi(x)]^{1-q}=(x-\gamma)^{q^{\frac{\alpha-\beta}{2}-1}}$ and $[\Psi(n)]^{1-p}=(n-\eta)^{\frac{\alpha-\beta}{2}-1}$.

Theorem 5 If $0<\alpha+\beta \leq 2, \gamma \in \mathbf{R}, \eta \leq 1-\frac{\alpha+\beta}{8}\left(1+\sqrt{3+\frac{4}{\alpha+\beta}}\right), p>1, \frac{1}{p}+\frac{1}{q}=1, f(x), a_{n} \geq 0$, $f \in L_{p, \Phi}(\gamma, \infty), a=\left\{a_{n}\right\}_{n=1}^{\infty} \in l_{q, \Psi},\|f\|_{p, \Phi}>0$ and $\|a\|_{q, \Psi}>0$, then we have the following 
equivalent inequalities:

$$
\begin{aligned}
I & :=\sum_{n=1}^{\infty} a_{n} \int_{\gamma}^{\infty} \frac{(\min \{1,(x-\gamma)(n-\eta)\})^{\beta}}{(\max \{1,(x-\gamma)(n-\eta)\})^{\alpha}} f(x) d x \\
& =\int_{\gamma}^{\infty} f(x) \sum_{n=1}^{\infty} \frac{(\min \{1,(x-\gamma)(n-\eta)\})^{\beta} a_{n}}{(\max \{1,(x-\gamma)(n-\eta)\})^{\alpha}} d x<\frac{4}{\alpha+\beta}\|f\|_{p, \Phi}\|a\|_{q, \Psi}, \\
J & =\left\{\sum_{n=1}^{\infty}[\Psi(n)]^{1-p}\left[\int_{\gamma}^{\infty} \frac{(\min \{1,(x-\gamma)(n-\eta)\})^{\beta} f(x)}{(\max \{1,(x-\gamma)(n-\eta)\})^{\alpha}} d x\right]^{p}\right\}^{\frac{1}{p}} \\
& <\frac{4}{\alpha+\beta}\|f\|_{p, \Phi}, \\
L & :=\left\{\int_{\gamma}^{\infty}[\Phi(x)]^{1-q}\left[\sum_{n=1}^{\infty} \frac{(\min \{1,(x-\gamma)(n-\eta)\})^{\beta} a_{n}}{(\max \{1,(x-\gamma)(n-\eta)\})^{\alpha}}\right]^{q} d x\right\}^{\frac{1}{q}} \\
& <\frac{4}{\alpha+\beta}\|a\|_{q, \Psi},
\end{aligned}
$$

where the constant $\frac{4}{\alpha+\beta}$ is the best possible in the above inequalities.

Proof The two expressions for $I$ in (18) follow from the Lebesgue term-by-term integration theorem. By (14) and (12), we have (19). By Hölder's inequality, we have

$$
\begin{aligned}
I & =\sum_{n=1}^{\infty}\left[\Psi^{\frac{-1}{q}}(n) \int_{\gamma}^{\infty} \frac{(\min \{1,(x-\gamma)(n-\eta)\})^{\beta} f(x)}{(\max \{1,(x-\gamma)(n-\eta)\})^{\alpha}} d x\right]\left[\Psi^{\frac{1}{q}}(n) a_{n}\right] \\
& \leq J\|a\|_{q, \Psi} .
\end{aligned}
$$

Then by (19), we have (18). On the other hand, assume that (18) is valid. Setting

$$
a_{n}:=[\Psi(n)]^{1-p}\left[\int_{\gamma}^{\infty} \frac{(\min \{1,(x-\gamma)(n-\eta)\})^{\beta} f(x)}{(\max \{1,(x-\gamma)(n-\eta)\})^{\alpha}} d x\right]^{p-1}, \quad n \in \mathbf{N}
$$

where $J^{p-1}=\|a\|_{q, \Psi}$. By (14), we find $J<\infty$. If $J=0$, then (19) is trivially valid; if $J>0$, then by (18) we have

$$
\|a\|_{q, \Psi}^{q}=J^{q(p-1)}=J^{p}=I<\frac{4}{\alpha+\beta}\|f\|_{p, \Phi}\|a\|_{q, \Psi},
$$

therefore $\|a\|_{q, \Psi}^{q-1}=J<\frac{4}{\alpha+\beta}\|f\|_{p, \Phi}$; that is, (19) is equivalent to (18). On the other hand, by (12) we have $[\varpi(x)]^{1-q}>\left(\frac{4}{\alpha+\beta}\right)^{1-q}$. Then in view of (15), we have (20). By Hölder's inequality, we find

$$
\begin{aligned}
I & =\int_{\gamma}^{\infty}\left[\Phi^{\frac{1}{p}}(x) f(x)\right]\left[\Phi^{\frac{-1}{p}}(x) \sum_{n=1}^{\infty} \frac{(\min \{1,(x-\gamma)(n-\eta)\})^{\beta} a_{n}}{(\max \{1,(x-\gamma)(n-\eta)\})^{\alpha}}\right] d x \\
& \leq\|f\|_{p, \Phi} L .
\end{aligned}
$$


Then by (20), we have (18). On the other hand, assume that (18) is valid. Setting

$$
f(x):=[\Phi(x)]^{1-q}\left[\sum_{n=1}^{\infty} \frac{(\min \{1,(x-\gamma)(n-\eta)\})^{\beta} a_{n}}{(\max \{1,(x-\gamma)(n-\eta)\})^{\alpha}}\right]^{q-1}, \quad x \in(\gamma, \infty),
$$

then $L^{q-1}=\|f\|_{p, \Phi}$. By (15), we find $L<\infty$. If $L=0$, then (20) is trivially valid; if $L>0$, then by (18), we have

$$
\|f\|_{p, \Phi}^{p}=L^{p(q-1)}=I<\frac{4}{\alpha+\beta}\|f\|_{p, \Phi}\|a\|_{q, \Psi},
$$

therefore $\|f\|_{p, \Phi}^{p-1}=L<\frac{4}{\alpha+\beta}\|a\|_{q, \Psi}$; that is, (20) is equivalent to (18). Hence, (18), (19) and (20) are equivalent.

If there exists a positive number $k\left(\leq \frac{4}{\alpha+\beta}\right)$ such that (18) is valid as we replace $\frac{4}{\alpha+\beta}$ with $k$, then, in particular, it follows that $\widetilde{I}<k \widetilde{H}$. In view of (16) and (17), we have

$$
\frac{(\alpha+\beta)(1-\eta)^{-\varepsilon}}{\left(\frac{\alpha+\beta}{2}\right)^{2}-\left(\frac{\varepsilon}{p}\right)^{2}}-\varepsilon O(1)<k\left[\varepsilon(1-\eta)^{-\varepsilon-1}+(1-\eta)^{-\varepsilon}\right]^{\frac{1}{q}},
$$

and $\frac{4}{\alpha+\beta} \leq k\left(\varepsilon \rightarrow 0^{+}\right)$. Hence, $k=\frac{4}{\alpha+\beta}$ is the best value of $(18)$.

By the equivalence of the inequalities, the constant factor $\frac{4}{\alpha+\beta}$ in (19) and (20) is the best possible.

Remark 1 (i) Define the first type half-discrete Hilbert-type operator $T_{1}: L_{p, \Phi}(\gamma, \infty) \rightarrow$ $l_{p, \Psi^{1-p}}$ as follows. For $f \in L_{p, \Phi}(\gamma, \infty)$, we define $T_{1} f \in l_{p, \Psi^{1-p}}$ by

$$
T_{1} f(n)=\int_{\gamma}^{\infty} \frac{(\min \{1,(x-\gamma)(n-\eta)\})^{\beta}}{(\max \{1,(x-\gamma)(n-\eta)\})^{\alpha}} f(x) d x, \quad n \in \mathbf{N}
$$

Then by (19), $\left\|T_{1} f\right\|_{p, \Psi^{1-p}} \leq \frac{4}{\alpha+\beta}\|f\|_{p, \Phi}$ and so $T_{1}$ is a bounded operator with $\left\|T_{1}\right\| \leq \frac{4}{\alpha+\beta}$. Since by Theorem 5 the constant factor in (19) is best possible, we have $\left\|T_{1}\right\|=\frac{4}{\alpha+\beta}$.

(ii) Define the second type half-discrete Hilbert-type operator $T_{2}: l_{q, \Psi} \rightarrow L_{q, \Phi^{1-q}}(\gamma, \infty)$ as follows. For $a \in l_{q, \Psi}$, we define $T_{2} a \in L_{q, \Phi^{1-q}}(\gamma, \infty)$ by

$$
T_{2} a(x)=\sum_{n=1}^{\infty} \frac{(\min \{1,(x-\gamma)(n-\eta)\})^{\beta}}{(\max \{1,(x-\gamma)(n-\eta)\})^{\alpha}} a_{n}, \quad x \in(\gamma, \infty) .
$$

Then by (20), $\left\|T_{2} a\right\|_{q, \Phi^{1-q}} \leq \frac{4}{\alpha+\beta}\|a\|_{q, \Psi}$ and so $T_{2}$ is a bounded operator with $\left\|T_{2}\right\| \leq \frac{4}{\alpha+\beta}$. Since by Theorem 5 the constant factor in (20) is best possible, we have $\left\|T_{2}\right\|=\frac{4}{\alpha+\beta}$.

Remark 2 (i) For $p=q=2$, (18) reduces to (6). Since we find

$$
\begin{aligned}
& \min _{0<\alpha+\beta \leq 2}\left\{1-\frac{\alpha+\beta}{8}\left(1+\sqrt{3+\frac{4}{\alpha+\beta}}\right)\right\} \\
& =\min _{0<\alpha+\beta \leq 2}\left\{1-\frac{\alpha+\beta}{8}-\frac{1}{8} \sqrt{3(\alpha+\beta)^{2}+4(\alpha+\beta)}\right\} \\
& =\frac{3-\sqrt{5}}{4}=0.19^{+}>0,
\end{aligned}
$$


then for $\eta=\gamma=0$ in (18), we have the following inequality:

$$
\begin{aligned}
& \sum_{n=1}^{\infty} a_{n} \int_{0}^{\infty} \frac{(\min \{1, x n\})^{\beta}}{(\max \{1, x n\})^{\alpha}} f(x) d x \\
& \quad<\frac{4}{\alpha+\beta}\left\{\int_{0}^{\infty} x^{p\left(1-\frac{\alpha-\beta}{2}\right)-1} f^{p}(x) d x\right\}^{\frac{1}{p}}\left\{\sum_{n=1}^{\infty} n^{q\left(1-\frac{\alpha-\beta}{2}\right)-1} a_{n}^{q}\right\}^{\frac{1}{q}} .
\end{aligned}
$$

Hence, (18) is a more accurate inequality of (21).

(ii) For $\beta=0$ in (18), we have $0<\alpha \leq 2, \gamma \in \mathbf{R}, \eta \leq 1-\frac{\alpha}{8}\left(1+\sqrt{3+\frac{4}{\alpha}}\right)$, and

$$
\begin{aligned}
& \sum_{n=1}^{\infty} a_{n} \int_{\gamma}^{\infty} \frac{f(x) d x}{(\max \{1,(x-\gamma)(n-\eta)\})^{\alpha}} \\
& \quad<\frac{4}{\alpha}\left\{\int_{\gamma}^{\infty}(x-\gamma)^{p\left(1-\frac{\alpha}{2}\right)-1} f^{p}(x) d x\right\}^{\frac{1}{p}}\left\{\sum_{n=1}^{\infty}(n-\eta)^{q\left(1-\frac{\alpha}{2}\right)-1} a_{n}^{q}\right\}^{\frac{1}{q}} ;
\end{aligned}
$$

for $\alpha=0$ in (18), we have $0<\beta \leq 2, \gamma \in \mathbf{R}, \eta \leq 1-\frac{\beta}{8}\left(1+\sqrt{3+\frac{4}{\beta}}\right)$, and

$$
\begin{aligned}
& \sum_{n=1}^{\infty} a_{n} \int_{\gamma}^{\infty}(\min \{1,(x-\gamma)(n-\eta)\})^{\beta} f(x) d x \\
& \quad<\frac{4}{\beta}\left\{\int_{\gamma}^{\infty}(x-\gamma)^{p\left(1+\frac{\beta}{2}\right)-1} f^{p}(x) d x\right\}^{\frac{1}{p}}\left\{\sum_{n=1}^{\infty}(n-\eta)^{q\left(1+\frac{\beta}{2}\right)-1} a_{n}^{q}\right\}^{\frac{1}{q}} ;
\end{aligned}
$$

for $\beta=\alpha=\lambda$ in (18), we have $0<\lambda \leq 1, \gamma \in \mathbf{R}, \eta \leq 1-\frac{\lambda}{4}\left(1+\sqrt{3+\frac{2}{\lambda}}\right)$, and

$$
\begin{aligned}
& \sum_{n=1}^{\infty} a_{n} \int_{\gamma}^{\infty}\left[\frac{\min \{1,(x-\gamma)(n-\eta)\}}{\max \{1,(x-\gamma)(n-\eta)\}}\right]^{\lambda} f(x) d x \\
& \quad<\frac{2}{\lambda}\left\{\int_{\gamma}^{\infty}(x-\gamma)^{p-1} f^{p}(x) d x\right\}^{\frac{1}{p}}\left\{\sum_{n=1}^{\infty}(n-\eta)^{q-1} a_{n}^{q}\right\}^{\frac{1}{q}} .
\end{aligned}
$$

\section{Competing interests}

The authors declare that they have no competing interests.

\section{Authors' contributions}

BY carried out the molecular genetic studies participated in the sequence alignment and drafted the manuscript. XL conceived of the study and participated in its design and coordination. All authors read and approved the final manuscript.

\section{Author details}

'Department of Mathematics, Guangdong University of Education, Guangzhou, Guangdong 510303, China. ${ }^{2}$ Department of Mathematics, Zhaoqing University, Zhaoqing, Guangdong 526061, China.

\section{Acknowledgements}

This work is supported by Guangdong Natural Science Foundation (No. 7004344). 


\section{References}

1. Hardy, GH, Littlewood, JE, Pólya, G: Inequalities. Cambridge University Press, Cambridge (1934)

2. Mitrinović, DS, Pečarić, JE, Fink, AM: Inequalities Involving Functions and Their Integrals and Derivatives. Kluwer Academic, Boston (1991)

3. Yang, B: Hilbert-Type Integral Inequalities. Bentham Science Publishers, Sharjah (2009)

4. Yang, B: Discrete Hilbert-Type Inequalities. Bentham Science Publishers, Sharjah (2011)

5. Yang, B: On Hilbert's integral inequality. J. Math. Anal. Appl. 220, 778-785 (1998)

6. Yang, B: The Norm of Operator and Hilbert-Type Inequalities. Science Press, Beijing (2009)

7. Yang, B, Brnetić, I, Krnić, M, Pečarić, J: Generalization of Hilbert and Hardy-Hilbert integral inequalities. Math. Inequal Appl. 8(2), 259-272 (2005)

8. Krnić, M, Pečarić, J: Hilbert's inequalities and their reverses. Publ. Math. (Debr.) 67(3-4), 315-331 (2005)

9. Jin, J, Debnath, L: On a Hilbert-type linear series operator and its applications. J. Math. Anal. Appl. 371, 691-704 (2010)

10. Azar, L: On some extensions of Hardy-Hilbert's inequality and applications. J. Inequal. Appl. 2008, 546829 (2008)

11. Yang, B, Rassias, T: On the way of weight coefficient and research for Hilbert-type inequalities. Math. Inequal. Appl. 6(4), 625-658 (2003)

12. Arpad, B, Choonghong, O: Best constant for certain multilinear integral operator. J. Inequal. Appl. 2006, 28582 (2006)

13. Kuang, J, Debnath, L: On Hilbert's type inequalities on the weighted Orlicz spaces, pacific. J. Appl. Math. 1(1), 95-103 (2007)

14. Zhong, W: The Hilbert-type integral inequality with a homogeneous kernel of $\lambda$-degree. J. Inequal. Appl. 2008, $917392(2008)$

15. Yang, B: A new Hilbert-type operator and applications. Publ. Math. (Debr.) 76(1-2), 147-156 (2010)

16. Li, Y, He, B: On inequalities of Hilbert's type. Bull. Aust. Math. Soc. 76(1), 1-13 (2007)

17. Yang, B: A mixed Hilbert-type inequality with a best constant factor. Int. J. Pure Appl. Math. 20(3), 319-328 (2005)

18. Yang, B: A half-discrete Hilbert's inequality. J. Guangdong Educ. Inst. 31(3), 1-7 (2011)

19. Yang, B, Chen, Q: A half-discrete Hilbert-type inequality with a homogeneous kernel and an extension. J. Inequal. Appl. 2011, 124 (2011). doi:10.1186/1029-242X-2011-124

20. Chen, Q, Yang, B: On a more accurate half-discrete Mulholland's inequality and an extension. J. Inequal. Appl. 2012, 70 (2012). doi:10.1186/1029-242X-2012-70

21. Yang, B: A new half-discrete Mulholland-type inequality with parameters. Ann. Funct. Anal. 3(1), 142-150 (2012)

22. Kuang, J: Applied Inequalities. Shangdong Science Technic Press, Jinan (2004)

23. Kuang, J: Introduction to Real Analysis. Hunan Education Press, Chansha (1996)

\section{Submit your manuscript to a SpringerOpen ${ }^{\circ}$ journal and benefit from:}

- Convenient online submission

Rigorous peer review

- Immediate publication on acceptance

Open access: articles freely available online

- High visibility within the field

- Retaining the copyright to your article 